\title{
Experimental Evidence for Soliton Explosions
}

\author{
Steven T. Cundiff, ${ }^{1, *}$ J. M. Soto-Crespo, ${ }^{2}$ and Nail Akhmediev ${ }^{3}$ \\ ${ }^{1}$ JILA, National Institute of Standards and Technology and University of Colorado, \\ Boulder, Colorado 80309-0440 \\ ${ }^{2}$ Instituto de Óptica, C.S.I.C., Serrano 121, 28006 Madrid, Spain \\ ${ }^{3}$ Australian Photonics CRC, Optical Science Centre, Research School of Physics Science and Engineering, \\ Institute of Advanced Studies, Australian National University, Canberra ACT 0200, Australia
}

(Received 31 May 2001; published 4 February 2002)

\begin{abstract}
We show, experimentally and numerically, that Ti:sapphire mode-locked lasers can operate in a regime in which they intermittently produce exploding solitons. This happens when the laser operates near a critical point. Explosions happen spontaneously, but external perturbations can trigger them. In stable operation, all explosions have similar features, but are not identical. The characteristics of the explosions depend on the intracavity dispersion.
\end{abstract}

DOI: $10.1103 /$ PhysRevLett.88.073903

The concept of solitons describes various physical phenomena ranging from solitary waves on a water surface to ultrashort optical pulses from a laser [1]. The main feature of solitons is that they propagate for a long time without visible changes. Mathematically, a soliton is a localized solution of a partial differential equation describing the evolution of a nonlinear system with an infinite number of degrees of freedom. Solitons are usually attributed to integrable systems. In this instance, solitons remain unchanged during soliton-soliton interactions, apart from a phase shift. They can be viewed as "modes" of the system, and, along with radiation modes, they can be used to solve initial-value problems using a nonlinear superposition [2]. Recently, the notion of solitons has been extended to systems that are not necessarily integrable. Particular interest for us is dissipative systems [3] because all physical systems are dissipative in reality. The effects of dissipation are particularly pronounced in long distance optical transmission lines [4] as well as in mode-locked lasers. Generation and propagation of such pulses can be described using the complex Ginzburg-Landau equation [5,6], the dispersion managed soliton approach [7], or a combination of the two. All these approaches reveal a number of unusual properties of dissipative solitons worthy of experimental confirmation.

Soliton dynamics in dissipative systems are much more complicated than in integrable systems. Dissipative dynamics depend drastically on the system parameters. The evolution can be periodic or chaotic or solitons can be switched from one stable state to another. Under certain circumstances, solitons have been predicted to undergo dramatic transients, but ultimately return to the original state [3]. In this Letter we report the experimental observation of temporal solitons undergoing such transients, which we dub "exploding solitons." During an explosion, the soliton energy and spectrum undergo dramatic changes, but return to the steady state value afterwards. The existence of such instabilities may impact the design of long distance communications systems and ultrafast
PACS numbers: 42.65.-k, 47.20.Ky

mode-locked lasers. Explosions are detrimental to either application; however, knowledge that they can occur and accurate, experimentally verified, models are required before systems can be designed to avoid them.

Mode-locked lasers provide an excellent test bed for soliton dynamics because they represent an essentially infinite propagation distance with identical conditions for each period. For example, polarization locked vector solitons were first observed in a mode-locked fiber laser [8]. The concept of soliton dispersion management yielded important insight into mode-locked laser dynamics [7]. From a practical point of view, stable ultrashort pulses are of interest. Dissipative systems admit stable pulses in a certain range of parameters. Beyond this range, pulses might change regularly or chaotically on propagation $[9,10]$. Experimentally, changes of the short pulses in shape or in length from one round-trip to another are often present in laser dynamics but usually are avoided.

One of the most striking forms of pulsations are pulse explosions that happen for certain values of the laser parameters. These were predicted theoretically [3] for dissipative systems but have not been previously observed. We have experimentally observed unstable "exploding" solitons in a Kerr lens mode-locked (KLM) Ti:sapphire laser [11]. To experimentally detect soliton explosions, we temporally record the output spectrum of the laser and the integrated energy of the pulse. Full characterization by a technique such as frequency resolved optical gating [12] would be preferable. However, all such techniques utilize optical nonlinearities and cannot make single shot measurements on a nanojoule pulse that is produced directly from a mode-locked oscillator. Thus, measurement of the temporally resolved spectrum is the only technique that can provide information about the pulse dynamics on time scales comparable to the round-trip time of the laser cavity.

The experimental setup is shown in Fig. 1. The laser has a "stretched" cavity that is 4 times longer than typical (40 ns round-trip time). The laser spectrum shows strong "Kelly" sidebands from phase matching of the dispersive 


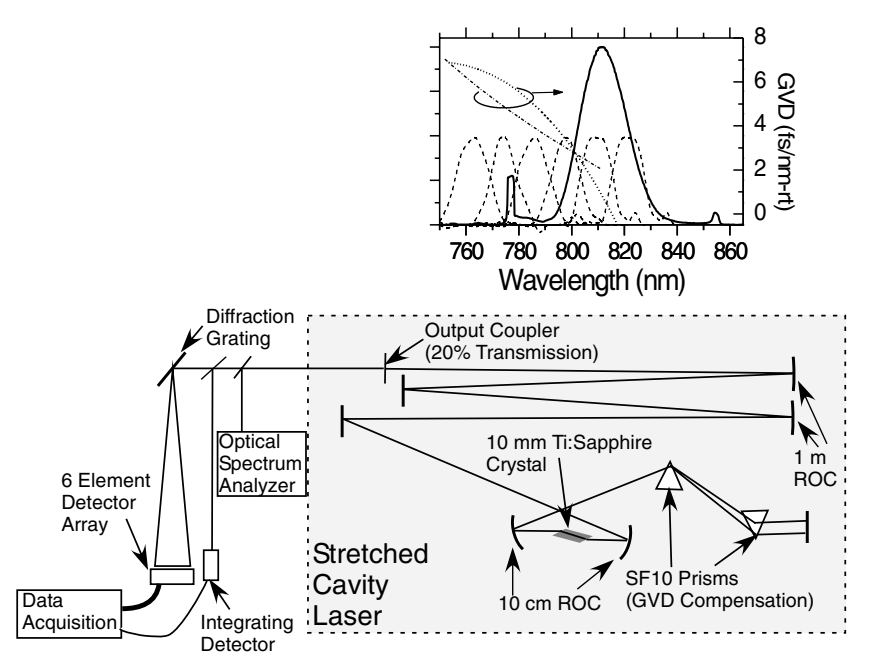

FIG. 1. Experimental setup. The radius of curvature (ROC) of the laser mirrors are indicated; those not designated are flat. The graph shows a typical steady state spectrum, the spectral response of the individual channels (dashed line), and the dispersion as a function of wavelength. The dotted dispersion curve corresponds to upper panel in Fig. 2 while the dot-dashed curve corresponds to the lower panel.

waves shed as the $50 \mathrm{fs}$ soliton undergoes periodic perturbation [13]. These are stronger than typically observed in a KLM laser due to the stretched cavity and lack of spectral filtering. The gain bandwidth is broader than the pulse spectrum and limited by the mirror reflectivity. The use of a stretched cavity is crucial because it means that the pulse spectrum is dominated by soliton dynamics through sideband formation, rather than through explicit spectral filtering due to a tuning element or mirror reflectivity. The output of the laser is spectrally dispersed by a diffraction grating across an array of six detectors. The spectral dispersion is adjusted so that each channel has a spectral width of $\sim 12 \mathrm{~nm}$ (full width at half maximum). The data are synchronously recorded with an electronic bandwidth corresponding to averaging over approximately five successive pulses.

The spectrally integrated intensity (i.e., total pulse energy) is also synchronously recorded on a separate channel. An optical spectrum analyzer is used to record the steady state spectrum. The six channels are positioned on the short wavelength side of the steady state spectrum because the spectral transients occur in that direction. This was verified by running the optical spectrum analyzer in "peak hold" mode during a large number of explosions. The long wavelength side of the captured spectrum was identical to the steady state spectrum. A typical steady state spectrum and the spectral response of the individual channels are shown in Fig. 1. Typical measured intracavity dispersion corresponding to the data in Fig. 2 is also shown. Measurements were performed in situ by measuring the repetition rate as a function of wavelength [14]. The change in slope and curvature show that higher order dispersion is present and changes between the two cases, hindering a simple quantitative assessment of the change.

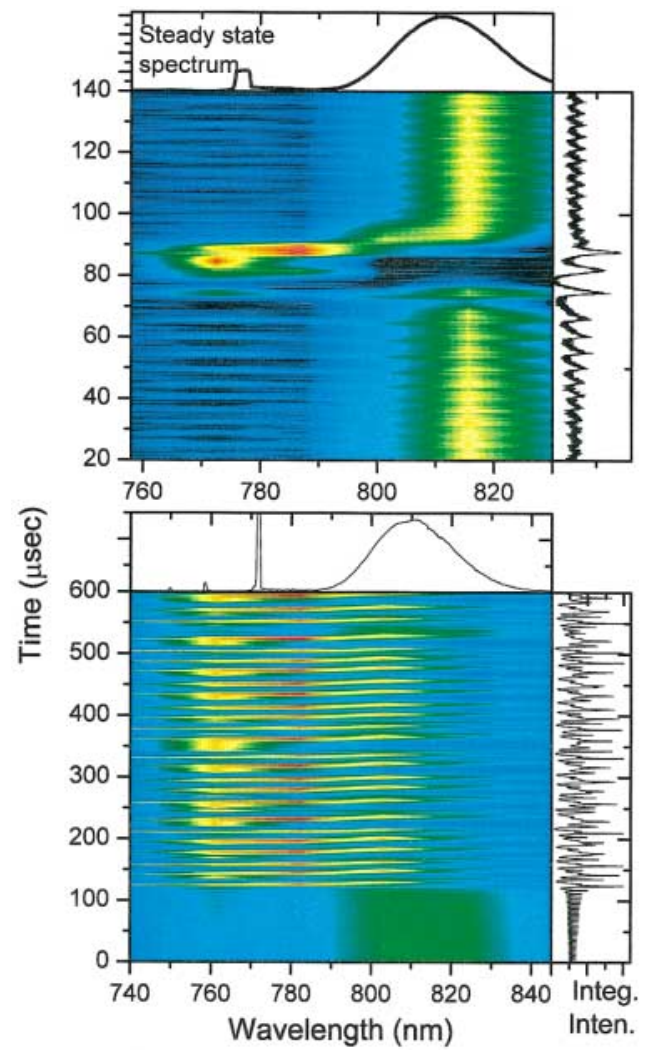

FIG. 2 (color). Typical data showing soliton explosions. In the upper panel the dispersion is adjusted to yield solitary explosions, while in the lower it is adjusted to yield bursts of explosions (see dispersion curves in Fig. 1). The resolution and position of spectral channels has been optimized in the lower panel.

Data exhibiting a typical soliton explosion are shown in Fig. 2. The bulk of the integrated spectrum is concentrated around the central frequency $812 \mathrm{~nm}$. When an explosion occurs, the spectrum becomes asymmetric, abruptly blueshifts, and subsequently returns to the original position. Small oscillations that slowly increase in amplitude precede an explosion and slowly die out after the explosion. Time averaged autocorrelations show that the steady state pulse is the same before and after an explosion and is transform limited. This fact, together with the observation that pulse spectrum narrows during the explosion, is proof that pulse becomes longer in time, as predicted [3], because of the Fourier transform limit.

The rate at which explosions occur can be controlled by changing the steady state soliton energy by adjusting the pump power. The range of pump powers for which explosions occur depends on the intracavity dispersion. A histogram of the frequency of the time between explosions ("laminar time") shows a power law dependence, with $P(\tau) \sim \tau^{-1}$. Strong external perturbations, such as a sharp rap on the laser, can trigger an explosion if the pump power is in the range for which explosions occur. No correlations with typical vibrations in the laboratory are observed. Thus the explosions are not simply due to technical noise. Although the onset of the explosions is 
not our focus here, power law behavior in the frequency of laminar times is a signature of "intermittency" in a system exhibiting chaotic dynamics [15].

If explicit spectral filtering is imposed on the laser, explosions do not occur, instead strong oscillations eventually break out at pump powers lower than those characteristic of the occurrence of explosions. We attribute these oscillations to relaxation phenomena because the damping time of relaxation oscillations increases as the pump power is lowered towards the lasing threshold [16]. The oscillations are distinct from explosions in that the large abrupt change in center wavelength does not occur; only the width and pulse energy oscillate.

All of the experimentally observed features are similar to those predicted theoretically in the continuous model [3]. However, the real system is not continuous. The discreteness of the laser must be taken into account to verify that the predictions still hold.

Numerical analysis has been done using a periodic system, similar to the experiment. Mode-locked lasers are typically modeled using the complex cubic-quintic Ginzburg-Landau (CGL) equation [6]:

$$
\begin{aligned}
i \psi_{z}+\frac{D}{2} \psi_{t t}+|\psi|^{2} \psi= & i \delta \psi+i \epsilon|\psi|^{2} \psi+i \beta \psi_{t t} \\
& +i \mu|\psi|^{4} \psi-\nu|\psi|^{4} \psi,
\end{aligned}
$$

where $t$ is retarded time (number of round trips), $z$ is the propagation distance, $\psi$ is the complex envelope of the electric field, $D$ is the dispersion coefficient, $\delta$ accounts for the linear gain, $\beta$ describes spectral filtering, $\epsilon$ and $\mu$ account for nonlinear gain/absorption processes, and $\nu$ is a possible higher order correction term to the intensitydependent refractive index.

We model the laser with the CGL equation but periodically vary the parameters $D, \delta, \beta, \epsilon, \mu$, and $\nu$ with $z$, the period corresponding to a cavity round-trip. The goal is to capture the essential effects of periodicity and abrupt changes of parameters inside the cavity, not to model the laser in detail. The map of the parameters is shown in the inset of Fig. 3. Dispersion, nonlinearity, and linear and nonlinear gain act for a propagation length, $L_{1}$ (modeling the evolution in the gain crystal). Concentrated losses at the end of a round-trip model the output coupler. For most of the cavity, the pulse propagates a distance $L_{2}$ under the sole effect of the dispersion term, $D_{2}$, the only important parameter in this stage being then the product $L_{2} D_{2}$. This accounts for the prisms induced dispersion. This model differs from the earlier work [3], which had distributed parameters. Explosions exist in the current model, which is closer to the experimental conditions.

Figure 3 illustrates the soliton explosions observed in the model. As the initial condition, we use a Gaussian pulse with the amplitude and width as parameters. This initial condition converges to a soliton with a shape that depends on the parameters. The soliton evolves and now and then explodes. Explosions happen irregularly but have

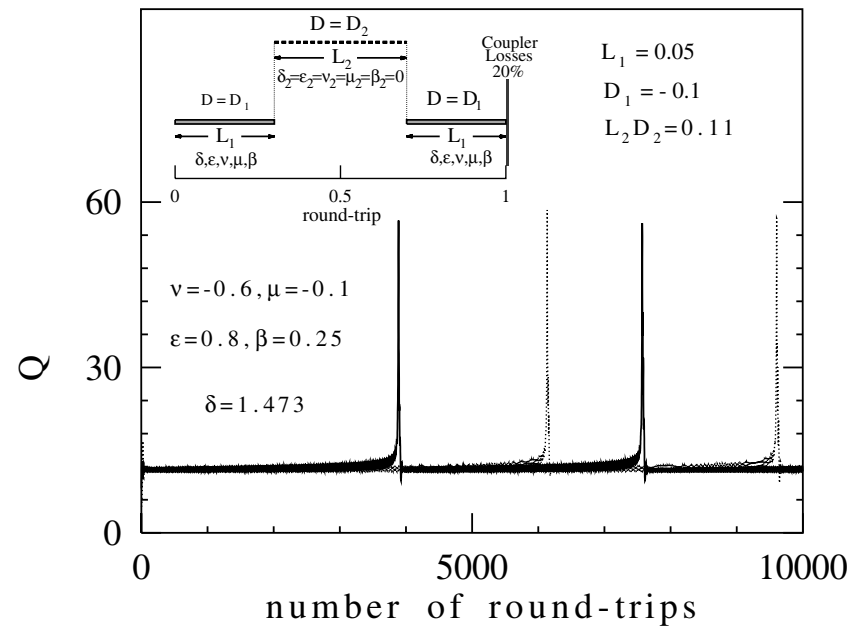

FIG. 3. Soliton explosions in numerical simulations. The soliton energy $Q$ is plotted versus the number of round-trips. $Q$ corresponds to the experimental integrated intensity. Parameters of the simulation are shown. The inset shows the map of the parameters of the CGLE for a single round-trip in the laser as used in our numerical simulations.

similar features. As in the experiment, oscillations with increasing amplitude precede the event. These oscillations die out quickly after each explosion. Changing initial conditions changes the time when any particular explosion happens. Figure 3 shows data for two different initial conditions. In each case, chaotic motion happens around the same localized structure. The number of explosions is indefinite in both cases. The oscillations around the explosion look very similar to the experiment. No attempt is made to model the Kerr-lens-mode-locking mechanism as it would require including the transverse dimensions to treat the self-focusing. This means that the transition to the "off" state is not accurately reproduced.

The pulse behavior depends on the model parameters. As in the experiment, the explosions appear at the edge of stability of stable pulse formation. In particular, the explosions are sensitive to the value of dispersion in both theory and experiment. For example, Fig. 4 shows that at $L_{2} D_{2}=0.0445$ the system generates stable solitons with very small pulsations ( $0.25 \%$ change in energy) with a period of approximately 15 round-trips. Increasing dispersion to $L_{2} D_{2}=0.0455$ increases the energy oscillations up to $5 \%$. Further increase of the dispersion up to $L_{2} D_{2}=$ 0.0457 results in explosions. When dispersion is increased to $L_{2} D_{2}=0.0459$, explosions happen more often. Similar effects are observed when other parameters are changed. Explosions occur in certain finite regions of the multidimensional parameter space. These regions can occupy appreciable regions of the parameter space [3].

We also calculated the spectral evolution of the pulse at the time of explosion. The spectra are symmetric in the quiet regime of soliton propagation. They become chaotic and asymmetric at the time of explosion. Third order dispersion increases this asymmetry. 

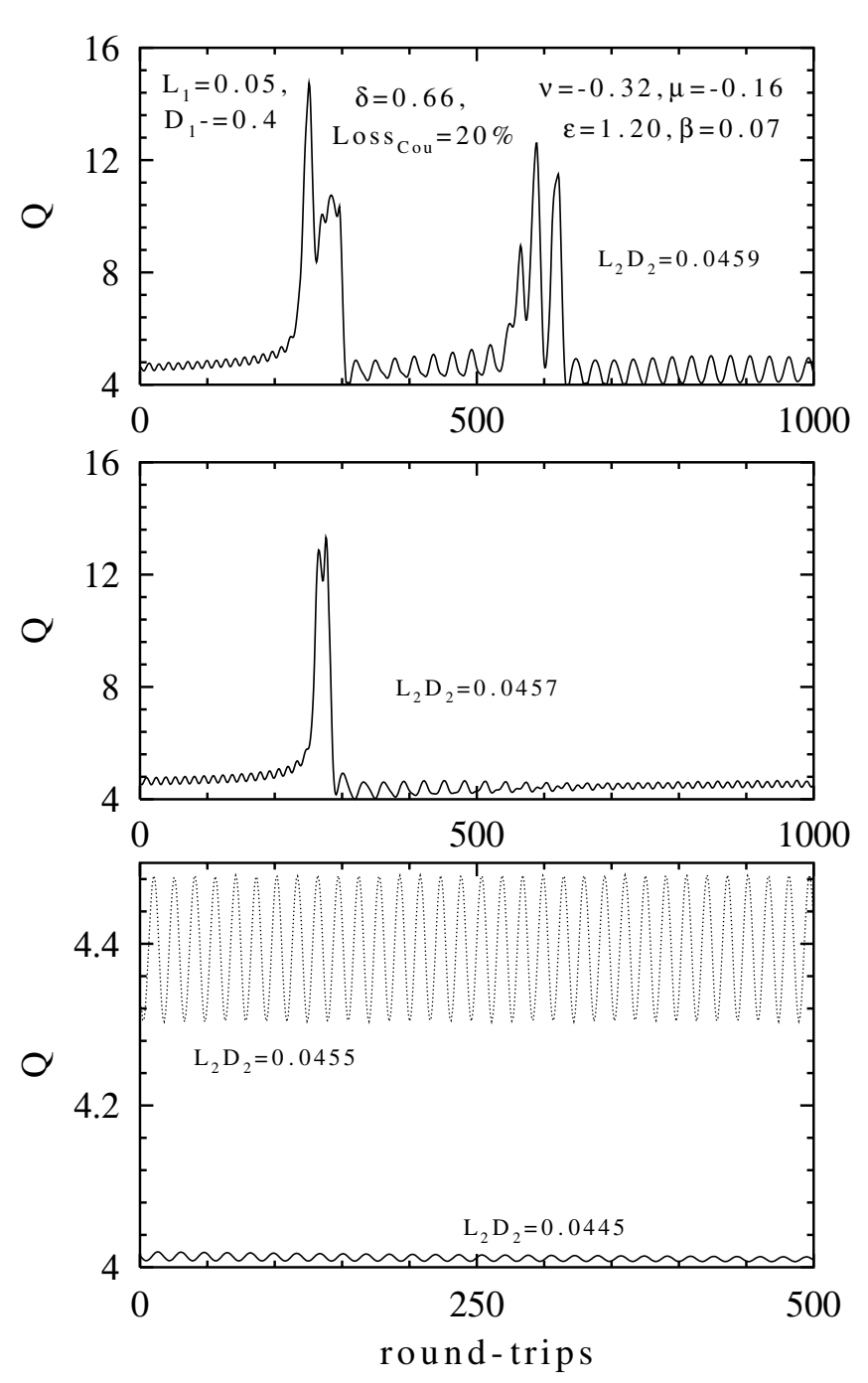

FIG. 4. Soliton energy versus the number of round-trips. Parameters of these simulations are shown in the figure. These results show that the occurrence of explosions is sensitive to $L_{2} D_{2}$ as in the experiment.

Finally, we note that oscillations observed in lasers are often ascribed to relaxation phenomena without further consideration due to their increased importance near lasing threshold [16]. However, our model displays oscillatory behavior, although it does not include a gain lifetime explicitly. Instead, these oscillations occur due to dynamic instability of the stationary solitons. Of course, in reality, oscillations occur due to both effects. Therefore we suggest that more care is needed in determining the origin of such oscillations. This may have important consequence in optimizing laser designs.

In conclusion, we have observed soliton explosions in a solid state passively mode-locked laser. To the best of our knowledge, this is the first observation of such soliton instabilities. The essential features of explosions, observed both experimentally and theoretically, are as follows: (1) Explosions occur occasionally. In the continuous model, they happen fairly regularly, but the period changes dramatically with a change of parameters. (2) The rate is very sensitive to average soliton energy (the pump power). (3) The laser is near a critical point close to unstable operation. (4) The explosions have similar features, but are not identical. (5) Explosions happen spontaneously, but external perturbations can trigger them. (6) There is no event preceding the onset of oscillations.

The work of J.M.S.C. was supported under Contract No. BFM2000-0806. S. T. C. acknowledges support from NIST and NSF. N.N.A. acknowledges support from U.S. AROFE (Grant No. N62649-01-1-0002).

Note added.-After submission of this manuscript, we have extended the model to include third order dispersion. This produces spectrally asymmetric explosions.

*Staff member, NIST Quantum Physics Division. Email address: cundiffs@jila.colorado.edu.

[1] See, for example, the focus issue of Chaos on "Optical Solitons" [Chaos 10 (2000)].

[2] V. E. Zakharov and A. B. Shabat, Sov. Phys. JETP 34, 62-69 (1971); for a textbook treatment, see M. J. Ablowitz and H. Segur, Solitons and the Inverse Scattering Transformation (SIAM, Philadelphia, 1981).

[3] J. M. Soto-Crespo, N. Akhmediev, and A. Ankiewicz, Phys. Rev. Lett. 85, 2937 (2000).

[4] A. Hasegawa and Y. Kodama, Solitons in Optical Communications (Oxford University Press, New York, 1995).

[5] H. Haus, J. Appl. Phys. 46, 3049 (1975).

[6] N. Akhmediev and A. Ankiewicz, Solitons, Nonlinear Pulses and Beams (Chapman \& Hall, London, 1997).

[7] Y. Chen et al., J. Opt. Soc. Am. B 16, 1999 (1999).

[8] S. T. Cundiff et al., Phys. Rev. Lett. 82, 3988 (1999).

[9] S. R. Bolton and M. R. Acton, Phys. Rev. A 62, 063803 (2000).

[10] N. Akhmediev, J. M. Soto-Crespo, and G. Town, Phys. Rev. E 63, 056602 (2001).

[11] For a review of passive mode-locking see, for example, E. P. Ippen, Appl. Phys. B 58, 159 (1994).

[12] R. Trebino et al., Rev. Sci. Instrum. 68, 3277 (1997).

[13] S. M. J. Kelly, Electron. Lett. 28, 806 (1992); J. P. Gordon, J. Opt. Soc. Am. B 9, 91 (1992).

[14] W. H. Knox, Opt. Lett. 17, 514 (1992).

[15] H. G. Schuster, Deterministic Chaos (VCH, Weinheim, 1995), 3rd ed.

[16] A.E. Siegman, Lasers (University Science Books, Mill Valley, 1986), pp. 962-964. 